\title{
ALTERAÇÃO DE MOBILIDADE DE PREGA VOCAL UNILATERAL: AVALIAÇÃO SUBJETIVA E OBJETIVA DA VOZ NOS MOMENTOS PRÉ E PÓS-FONOTERAPIA
}

\author{
Unilateral vocal fold mobility alteration: objective and subjective \\ evaluation of voice quality on prior and post speech therapy
}

Ana Cristina Cortes Gama ${ }^{(1)}$, Amanda Peixoto Faria ${ }^{(2)}$, Iara Barreto Bassi (3), Sônia Silvia Diniz (4)

\section{RESUMO}

Objetivo: avaliar de forma subjetiva e objetiva a voz de pacientes com paralisia unilateral de prega vocal nos momentos pré-tratamento e pós-tratamento. Método: trata-se de um estudo retrospectivo por meio de revisão de prontuário, que analisou as gravações de vozes de 12 indivíduos com diagnóstico otorrinolaringológico de paralisia unilateral de prega vocal. O material de voz colhido foi a emissão sustentada da vogal /a/, seguida de fala encadeada. As vozes pré e pós-terapia foram analisadas por meio da escala GRBASI, análise espectrográfica e medida do tempo máximo de fonação (TMF). Os parâmetros para análise espectrográfica foram: forma do traçado, grau de escurecimento dos harmônicos, continuidade do traçado, presença de ruídos, presença de sub-harmônicos e harmônicos definidos. A medida do TMF da vogal /a/ representou a maior de três emissões. Os dados obtidos foram submetidos a análise descritiva de tendência central e dispersão, e ao Teste Wilcoxon. Resultados: na análise perceptivo-auditiva, o parâmetro que mais se modificou no momento póstratamento foi o de soprosidade $(B)(p=0,003)$, seguido do grau da disfonia $(G)(p=0,004)$ e astenia (A) $(p=0,01)$, sendo que estes resultados foram estatisticamente significantes. Com relação ao espectrograma, houve melhora do traçado em $91 \%$ dos pacientes, e os parâmetros que mais se modificaram foram: aumento do número de harmônicos (32\%) e diminuição do ruído (24\%). A medida do TMF da vogal /a/ apresentou-se significantemente maior no momento pós-fonoterapia ( $p=0,003 \%$ ). Conclusão: pacientes com paralisia de prega vocal que foram submetidos ao tratamento fonoaudiológico apresentaram melhora dos dados perceptivo-auditivos, espectrográfico e do TMF.

DESCRITORES: Voz; Distúrbios da Voz; Fonoterapia; Paralisia das Pregas Vocais, Acústica da Fala

\section{INTRODUÇÃO}

As paralisias de pregas vocais são decorrentes de variadas causas, podendo ser sintomas

(1) Fonoaudióloga; Professora Adjunto do Departamento de Fonoaudiologia da Universidade Federal de Minas Gerais/ UFMG, Belo Horizonte - MG.

(2) Fonoaudióloga graduada pela Universidade Federal de Minas Gerais/UFMG, Belo Horizonte - MG.

(3) Fonoaudióloga; Mestranda em Saúde e Trabalho pelo Programa de Pós-graduação em Saúde Pública do Departamento de Medicina Preventiva e Social da Faculdade de Medicina da Universidade Federal de Minas Gerais UFMG - Belo Horizonte (MG), Brasil.

(4) Fonoaudióloga graduada pela Universidade Federal de Minas Gerais/UFMG, Belo Horizonte - MG.

Conflito de interesses: inexistente secundários de outras patologias, incluindo patologias do Sistema Nervoso Central ou Periférico, traumas mecânicos de pescoço, cabeça e tórax, hipertrofia cardíaca, neoplasias, causas cirúrgicas, tóxicas, metabólicas, inflamatórias e idiopáticas ${ }^{1-6}$. Diversas posições podem ser assumidas pelas pregas vocais paralisadas, e podem ser classificadas como mediana, para-mediana, intermediária, em abdução e em abdução forçada ou cadavérica $^{1,3,7}$.

Quando ocorre bilateralmente a paralisia de pregas vocais apresenta-se tipicamente acompanhada de obstrução de via aérea. A voz pode apresentar-se aguda ou razoavelmente boa, devido à posição paramediana de ambas as pregas. Muitas vezes pode configurar uma emergência respiratória, 
podendo requerer entubação ou traqueostomia urgente $^{1,8}$. Nos casos de paralisia unilateral de prega vocal, observa-se tipicamente disfagia, incoordenação pneumofonoarticulatória, fadiga vocal, rouquidão, redução da intensidade, redução da extensão vocal, perda da projeção vocal e soprosidade na voz, cujo grau de severidade pode manifestar-se de acordo com o grau de incompetência glótica $^{1,8,9}$.

Esta, por sua vez, depende da posição relativa da prega vocal paralisada (PVP) e da habilidade da prega contra lateral em compensar o fechamento glótico, cruzando a linha média da glote. A paralisia unilateral de prega vocal é comumente encontrada na prática clínica, embora sua incidência permaneça desconhecida ${ }^{3}$. Esses casos podem apresentar disfagia orofaríngea associada a mudanças nas medidas acústicas e perceptivo-auditivas da voz tais como intensidade, extensão vocal, frequência fundamental e o tempo máximo de fonação (TMF) diminuídos ${ }^{3,10}$.

Nas paralisias unilaterais de prega vocal a conduta de eleição é a fonoterapia, que apresenta por objetivo eliminar os riscos de aspiração de alimentos e saliva e melhorar a qualidade vocal. As técnicas utilizadas têm por objetivo melhorar a coaptação glótica, aproximando as pregas vocais ${ }^{3,10-13}$. A terapia vocal, que muitas vezes pode ser realizada como pré-operatória ou evidenciar a necessidade de intervenção cirúrgica, ajuda o paciente quanto a decisões cirúrgicas pendentes e fornece o treinamento para a adequada fonação pós-operatória ${ }^{8}$.

A análise perceptivo-auditiva é um teste subjetivo que se baseia especialmente na impressão do avaliador sobre a voz, sofrendo influência do nível de experiência do mesmo neste tipo de avaliação, do material de voz analisado, do tipo de apresentação, do grau de desvio da qualidade vocal, além do tipo de escala utilizada nesta tarefa ${ }^{14}$.

Enquanto a avaliação perceptivo-auditiva oferece uma descrição do sinal sonoro tendo como instrumento apenas a audição, a avaliação acústica realiza a mensuração do sinal vocal, o que leva a uma análise mais objetiva. O espectrograma, obtido por meio da espectrografia acústica, é um gráfico que mostra uma sucessão de espectros unitários, em um registro tridimensional, que apresenta no eixo horizontal o tempo, a frequência no eixo vertical e a intensidade no grau de escurecimento das marcas do registro ${ }^{15}$. Os principais dados obtidos com o espectrograma são as características temporais da onda sonora.

O TMF é obtido por meio de teste rotineiramente aplicado em pacientes disfônicos, com intuito de avaliar a eficiência glótica, sendo os valores normais esperados para homens de 20 segundos e para mulheres de 15 segundos. Esses valores, na presença de paralisia de prega vocal, comumente encontram-se diminuídos, apresentando-se em torno de quatro a cinco segundos, o que leva a necessidade de recarga aérea a cada um ou dois segundos, provocando fadiga e hiper-ventilação ${ }^{9}$. A incompetência glótica causada pela paralisia de prega vocal provoca impacto não somente no sinal vocal, mas no sinal de fala como um todo ${ }^{16}$, verificando-se soprosidade, devido ao escape glótico e rouquidão, devido à vibração irregular das pregas vocais ${ }^{10}$.

Muitas são as informações na literatura relacionadas aos procedimentos cirúrgicos ${ }^{3,8,11,17}$, porém informações relacionadas ao efeito da terapia vocal para paralisia unilateral de prega vocal ainda são escassos. Torna-se, portanto, necessária a avaliação dos benefícios da terapia fonoaudiológica sobre os aspectos vocais relacionados às características perceptivo-auditivas e espectográficas.

O presente estudo tem como objetivo analisar as características perceptivo-auditivas e espectrográficas da voz em pacientes com imobilidade de prega vocal unilateral nos momentos pré-tratamento e pós-tratamento fonoaudiológico.

\section{MÉTODO}

Trata-se de um estudo retrospectivo por meio de revisão de prontuário, onde foram coletados dados de 12 pacientes com diagnóstico médico de paralisia unilateral de prega vocal, por exame de imagem estroboscópica com ausência de mobilidade de prega vocal durante a fonação. Todos os participantes foram atendidos por um mesmo profissional, em consultório particular de Belo Horizonte, no período de fevereiro de 2006 a dezembro de 2008. Os critérios de inclusão para a amostra foram: ter realizado fonoterapia; ter diagnóstico otorrinolaringológico de paralisia unilateral de prega vocal; ter tido sua voz registrada na primeira consulta e na alta fonoaudiológica.

De cada paciente foram coletados os seguintes dados: qual prega vocal foi paralisada; qual posição foi assumida pela prega paralisada; número de sessões realizadas de fonoterapia; TMF antes e após a terapia para reabilitação vocal; além de gravações da voz do paciente.

As gravações coletadas constaram da vogal /a/ sustentada, seguida de fala encadeada, que se tratou da emissão dos dias da semana. O mesmo procedimento foi realizado durante a avaliação da voz e após a reabilitação vocal, resultando, portanto em duas amostras de voz, correspondentes respectivamente às situações pré e pós-terapia fonoaudiológica. 
A gravação das vozes foi realizada diretamente em computador, equipado com microfone profissional, do tipo condensador, estéreo, omni-direcional, sensibilidade de $-20 \mathrm{~dB}$, da marca EQUITEK E-100, ligado a uma fonte de eletricidade (Phanton Power) de uma mesa de som marca MACKIE 1202 VLZ - 12 canais. Os indivíduos estavam em pé, com o microfone situado a $10 \mathrm{~cm}$ da boca e com ângulo de captação direcional de $90^{\circ}$. O microfone estava deslocado do corpo da unidade de gravação para evitar captação de ruído do maquinário. As gravações foram realizadas em ambiente silente.

A gravação da vogal sustentada e fala encadeada foi realizada em um PC IBM Aptiva E30P, processador $\mathrm{AMD}-\mathrm{K} 6-2$ / $500 \mathrm{MHz}$, memória de 128 mega bytes RAM, espaço de disco 8,4 Giga bytes, placa de som Crystal Sound Fusion.

As emissões foram analisadas acusticamente pelo programa Gram 5.0, o qual gera a imagem espectrográfica do som. Estas análises foram geradas em mono, sample rate de $11 \mathrm{k}$ e resolução de 16 bit e as espectrografias, geradas em escala de $60 \mathrm{~dB}$ em display scroll e paleta CB. A análise das frequências foi realizada em escala linear, banda estreita, FFT 1024 e resolução $5.4 \mathrm{~Hz}$.

Foram convidados a participar desta pesquisa cinco fonoaudiólogos que realizaram análise visualespectrográfica e avaliação perceptivo-auditiva das vozes. Para a análise perceptivo-auditiva utilizou-se a escala GRBASI, elaborada pela Sociedade Japonesa de Logopedia e Foniatria (Hirano, 1981) ${ }^{18} \mathrm{e}$ modificado por Dejonckere e Leback 1996 ${ }^{19}$. Esta escala é composta por seis parâmetros perceptivoauditivos: $(G)$ grau geral da disfonia, $(R)$ ruído, $(B)$ soprosidade, (A) astenia, (S) tensão e (I) instabilidade. Para cada parâmetro, o grau de alteração foi definido por uma escala análogico-visual, composta por uma régua de 10 centímetros, onde o extremo esquerdo significava ausência de alteração e o extremo direito o grau máximo de alteração.

Para a avaliação do traçado espectrográfico utilizou-se o protocolo proposto por $^{20}$, onde foram avaliados os seguintes parâmetros: forma do traçado, grau de escurecimento dos harmônicos, continuidade do traçado, presença de ruídos, presença de sub-harmônicos e harmônicos definidos.

Para a análise da concordância intra-sujeitos da avaliação perceptivo-auditiva, $20 \%$ da amostra foi repetida, totalizando 15 emissões para cada momento, a saber: pré e pós-fonoterapia. O mesmo procedimento foi realizado para a análise da concordância intra-sujeitos na análise preceptivo-visual do traçado espectrográfico.

A ordem da apresentação das 30 gravações para análise perceptivo-auditiva foi determinada aleatoriamente, por sorteio. A apresentação das vozes aos fonoaudiólogos foi feita com a utilização de fones de ouvido Sennheiser HD437 e computador Acer 4420-5239 AMD Athlon X2.

$\mathrm{Na}$ avaliação acústica os espectrogramas foram apresentados sequencialmente, e organizados de maneira aleatória, portanto, o avaliador não tinha conhecimento prévio se o espectro analisado era pré ou pós-tratamento. Os avaliadores deveriam analisar se o segundo espectrograma melhorou, piorou ou permaneceu estável em relação ao primeiro e, se uma das duas primeiras alternativas fosse escolhida, deveriam marcar dois parâmetros espectrográficos que correspondessem ao resultado encontrado.

Foi realizado teste de Spearman para encontrar a concordância intra-avaliador na avaliação perceptivo-auditiva e posteriormente, foram selecionados três dos cinco avaliadores que obtiveram os melhores coeficientes de correlação $(0,935$; $0,886 ; 0,829)$ para que constituíssem os resultados da pesquisa. Para se obter a concordância intraavaliador na avaliação espectrográfica foi utilizado o índice estatístico Kappa e encontrados três dos cinco avaliadores, que obtiveram os melhores coeficientes de correlação $(1,000 ; 0,847 ; 0,833)$.

Para a medida do TMF foi solicitado aos pacientes que emitissem a vogal /a/ o mais prolongado possível, no tom e intensidade habituais, por três vezes, sendo considerado o maior valor dentre as três medidas.

Este trabalho foi analisado pelo Comitê de Ética em Pesquisa (COEP) da Universidade Federal de Minas Gerais e aprovado com o número ETIC 222/08. Todos os convidados concordaram em participar da pesquisa assinando o "Termo de Consentimento Livre e Esclarecido".

Para avaliar as variações dos parâmetros da escala GRBASI e do TMF nos momentos pré e pósfonoterapia, foi utilizado o teste não paramétrico Wilcoxon. Adotado o nível de significância de 0,05. Para a escala espectrográfica foi realizada análise descritiva.

\section{RESULTADOS}

A pesquisa foi realizada com 12 pacientes, sendo 3 homens (25\%) e 9 mulheres $(75 \%)$, com idade entre 32 a 74 anos e média de 55 anos. Dentre estes, 9 indivíduos (67\%) tiveram a prega vocal esquerda paralisada e 3 a direita (33\%); sendo 7 pacientes com PVP em posição mediana $(58,3 \%)$ e 5 em posição para-mediana $(41,7 \%)$. O número de sessões fonoaudiológicas variou de seis a 33 sessões, com uma média de 11,54 sessões. 
Os resultados da análise perceptivo-auditiva e acústica estão dispostos nas tabelas 1, 2 e 3 . $\mathrm{Na}$ Tabela 1 estão distribuídos os valores médios obtidos na avaliação das vozes nos momentos pré e pós intervenção fonoaudiológica por meio da escala GRBASI. Observa-se que os parâmetros que mais se modificaram no momento pós- tratamento foram os de soprosidade (B) e grau da disfonia $(G)$, sendo que estes resultados foram estatisticamente significantes.

Tabela 1 - Distribuição dos valores de média e desvio padrão dos parâmetros da análise perceptivoauditiva nos momentos pré e pós-fonoterapia

\begin{tabular}{|c|c|c|c|c|c|}
\hline \multirow{2}{*}{ GRBASI } & \multicolumn{2}{|c|}{ Média } & \multicolumn{2}{|c|}{ Desvio Padrão } & \multirow{2}{*}{ Valor de $p$} \\
\hline & Pré & Pós & Pré & Pós & \\
\hline$G$ & 7,60 & 4,35 & 2,18 & 2,57 & $0,004^{*}$ \\
\hline $\mathrm{R}$ & 2,44 & 2,36 & 2,48 & 2,33 & 0,480 \\
\hline B & 6,30 & 2,34 & 2,75 & 1,85 & $0,003^{*}$ \\
\hline A & 1,63 & 0,38 & 1,25 & 0,61 & $0,010^{*}$ \\
\hline$S$ & 1,50 & 1,49 & 2,09 & 2,00 & 0,410 \\
\hline । & 2,35 & 1,80 & 1,28 & 1,52 & 0,136 \\
\hline
\end{tabular}

Teste de Wilcoxon

$p<0,05$

Na Tabela 2 estão os resultados da análise espectrográfica comparando-se os momentos pré e pós-fonoterapia. Os parâmetros da análise espectrográfica que melhoraram no momento pós-fonoterapia estão descritos na Tabela 3. Houve melhora do traçado espectrográfico em $91 \%$ dos pacientes (Figuras 1,2), e os parâmetros que mais se modificaram foram: aumento do número de harmônicos (32\%) e diminuição do ruído (24\%).

Tabela 2 - Resultado da análise do traçado espectrográfico comparando-se os momentos pré e pós-fonoterapia

\begin{tabular}{cccccc}
\hline \multirow{3}{*}{ Resultado } & \multicolumn{3}{c}{ Melhorou } & \multicolumn{2}{c}{ Estável } \\
\cline { 2 - 5 } & $\mathbf{N}$ & $\%$ & $\mathbf{N}$ & $\%$ \\
\cline { 2 - 6 } & 33 & 91,7 & 03 & 08,3 \\
\hline
\end{tabular}

Tabela 3-Distribuição dos parâmetros espectrográficos que melhoraram no momento pós-fonoterapia

\begin{tabular}{lcc}
\hline Parâmetro & N & $\%$ \\
\hline Harmônicos definidos & 32 & 32,3 \\
Presença de ruído & 24 & 24,2 \\
Forma do Traçado & 15 & 15,2 \\
Continuidade do traçado & 13 & 13,1 \\
Grau de escurecimento & 11 & 11,1 \\
Presença de sub-harmônicos & 4 & 4,1 \\
\hline
\end{tabular}




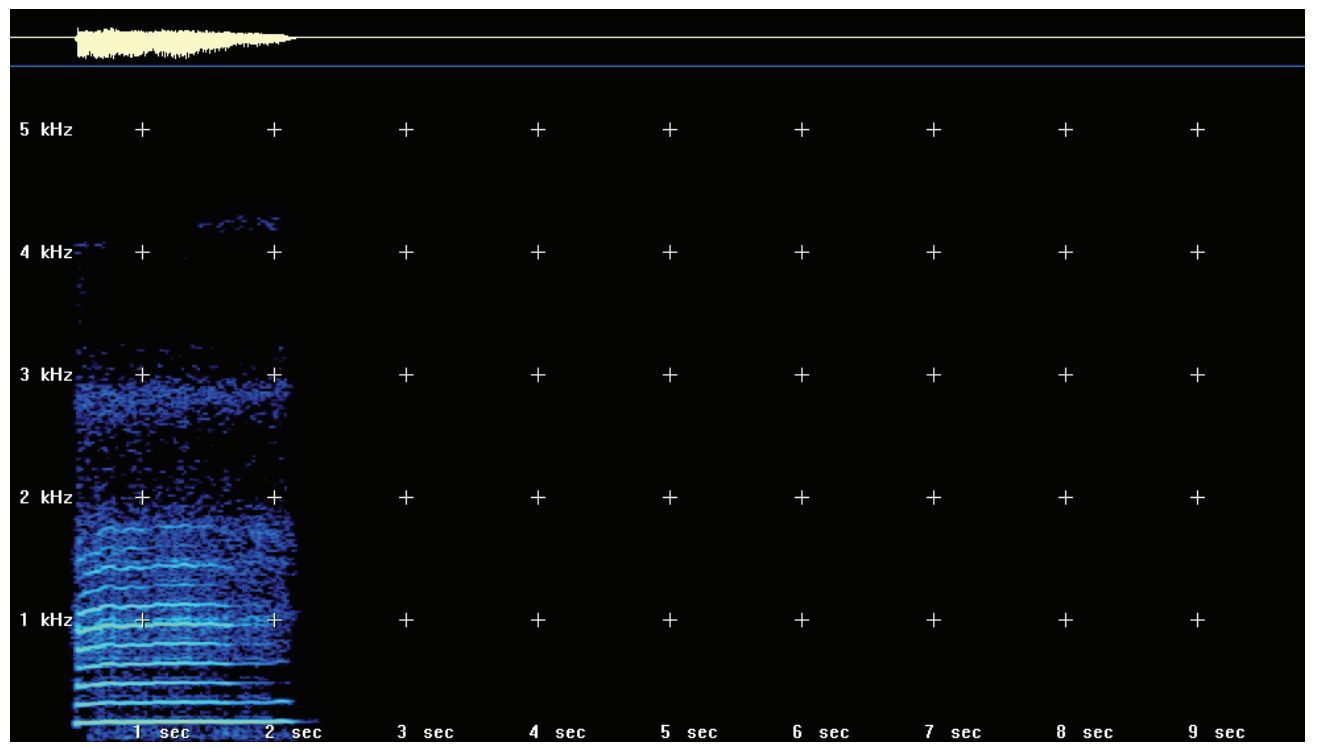

Figura 1 - Imagem de um espectrograma no momento pré-fonoterapia

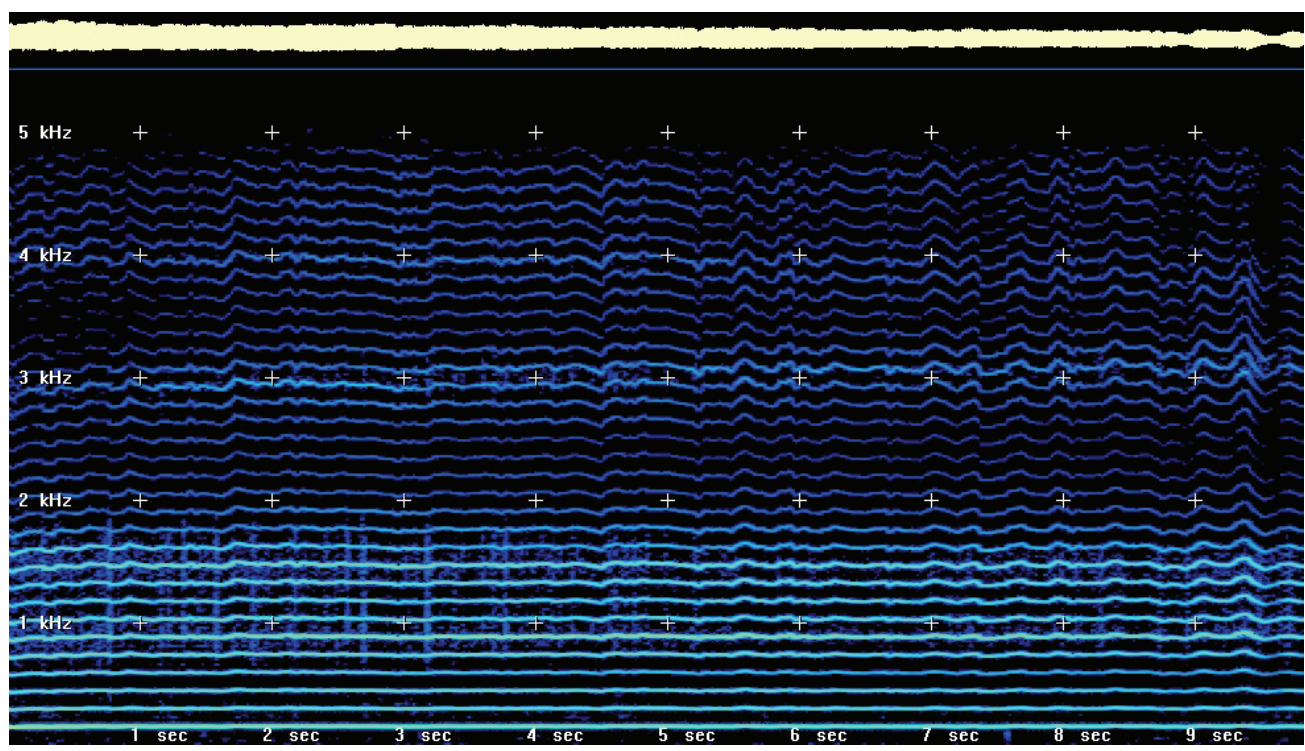

Figura 2 - Imagem de um espectrograma no momento pós-fonoterapia

Na Tabela 4 estão descritas as diferenças nos valores médios dos TMF nos momentos pré e pós-fonoterapia e a medida do TMF da vogal /a/ apresentou-se significantemente maior no momento pós-fonoterapia, diferença esta com significância estatística $(p=0,003)$.

Tabela 4 - Valores de média e desvio padrão do tempo máximo de fonaçao da vogal /A/ nos momentos pré e pós-fonoterapia

\begin{tabular}{cccc}
\hline & Média & Desvio padrão & Valor de $\mathbf{p}$ \\
\cline { 2 - 4 } Pré & 4,99 & 3,53 & \\
Pós & 9,35 & 3,84 & \multirow{2}{*}{$0,003^{\star}$} \\
Pós - pré & & & \\
\hline
\end{tabular}

Teste de Wilcoxon

$p<0,05$ 


\section{DISCUSSÃO}

Paralisia significa a perda da capacidade de movimentos voluntários de um músculo por efeito de lesão ou até mesmo doença que afete a unidade motora e o sistema nervoso central. As paralisias laríngeas podem ocorrer por dano periférico dos nervos laríngeos: nervo laríngeo superior (NLS) e nervo laríngeo inferior (NLI), ou por envolvimento do sistema nervoso central. As causas mais comuns podem ser congênita, iatrogênica, idiopática, neurológica e viral21. A causa mais frequente de paralisia unilateral das pregas vocais é a traumática, incluindo os traumatismos secundários às cirurgias cervicais como tireoidectomias, intubação traqueal, toracotomia, acidentes automobilísticos e ferimentos por armas. Causas inflamatórias de origem viral e patologias centrais são etiologias menos freqüentes ${ }^{22,23}$.

A avaliação do paciente com paralisia unilateral de prega vocal requer uma investigação detalhada e análise critica dos seus resultados. Deve ainda contar com exames videolaringoscópicos, avaliações objetivas e subjetivas da voz , $^{3,921-25}$.

Nos últimos anos, a eletromiografia da laringe vem sendo útil no diagnóstico das alterações de mobilidade de pregas vocais. Sobretudo diferenciando as paralisias das fixações e também fornecendo dados para o prognóstico e localização de lesões neuromusculares, sendo um método objetivo de avaliação da atividade neuromuscular ${ }^{26}$. No presente estudo, o diagnóstico de paralisia de prega vocal foi obtido em avaliação otorrinolaringológica por meio de exame de imagem verificando-se ausência de mobilidade de prega vocal à fonação. Apesar da eletromiografia de laringe ser a avaliação de eleição no diagnóstico diferencial das alterações de mobilidade de pregas vocais, não foi possível a realização do mesmo nos pacientes desta pesquisa pela dificuldade de acesso dos pacientes a tal procedimento.

Com relação à caracterização dos pacientes deste estudo, a faixa etária de maior ocorrência foi a idade adulta. Tal resultado vai ao encontro dos achados de um estudo retrospectivo de três anos que avaliou 117 pacientes e encontrou uma maior ocorrência da paralisia de prega vocal unilateral entre os 50 a 70 anos $^{23}$. O gênero mais prevalente desta pesquisa foi o feminino $(75 \%)$ e, tal resultado é divergente na literatura, encontrando ora amostras ligeiramente superiores de homens ${ }^{23}$ ora de mulheres ${ }^{21,25}$.Tais discordâncias podem ser justificadas pela multicausalidade da paralisia de prega vocal unilateral e também, pelo número reduzido de sujeitos das pesquisas.
Os casos de paralisia unilateral de prega vocal se apresentam em maior frequência no acometimento do lado esquerdo. Isto pode ser explicado devido ao fato do nervo laríngeo recorrente esquerdo apresentar trajeto mais longo e por sua relação com o ducto arterioso, tornando-o susceptível a injúrias no decorrer da evolução de várias doenças ou durante procedimentos cirúrgicos ${ }^{27}$. Um estudo encontrou a prevalência de $69,2 \%$ dos casos com paralisia unilateral de prega vocal à esquerda ${ }^{22}$. O presente estudo confirma esse dado, apresentando $67 \%$ dos pacientes com a prega vocal esquerda acometida.

As posições assumidas pela PVP têm sido objeto de numerosas publicações e controversas especulações se reportam às diferentes posições assumidas pela PVP ${ }^{10-16}$. As configurações glóticas adotadas na paralisia de prega vocal unilateral são influenciadas por diversos fatores que interagem entre si simultaneamente de uma forma complexa e há sempre o fator subjetivo do examinador ${ }^{28}$. $O$ presente estudo analisou 7 pacientes com PVP em posição mediana $(58,3 \%)$ e 5 em posição para-mediana (41,7\%). Esses dados concordam com o resultado de uma pesquisa realizada com 86 indivíduos com paralisia de prega vocal unilateral na qual $59 \%$ da amostra apresentava posição paramediana ${ }^{9}$.

O número de sessões de fonoterapia desta pesquisa foi, em média, de 11,54 sessões, mostrando valores similares com outros trabaIhos, com um tempo médio de 5,6 sessões ${ }^{23}$ e 12,6 sessões $^{25}$ de tratamento fonoaudiológico, o que permite sugerir que as alterações vocais decorrentes deste tipo de paralisia de prega vocal, apresentam um tempo de tratamento fonoaudiológico razoavelmente breve.

A avaliação perceptivo-auditiva da função vocal iniciou-se no século XIX, com a análise subjetiva da voz. Esta prática fonoaudiológica tem sido muito utilizada ainda nos dias de hoje para detectar alterações, buscando-se um equilíbrio do que se vê e se ouve do sujeito para análise e interpretação dos achados. A escala GRBASI é apontada como a avaliação perceptivo-auditiva mais utilizada e fornece informações importantes sobre a produção vocal e o grau da alteração ${ }^{27,29}$.

$\mathrm{Na}$ avaliação perceptivo-auditiva da voz, os valores da escala GRBASI que apresentaram melhora mais evidente após fonoterapia foram a soprosidade (B), o grau geral (G) e astenia (A) (Tabela 1). Os achados do presente estudo concordam com a pesquisa que avaliou a voz de 30 indivíduos, do sexo feminino, com paralisia unilateral de prega vocal, antes e após a terapia fonoaudiológica e encontrou que os valores médios para GRBASI foram reduzidos após a terapia 
fonoaudiológica ${ }^{25}$. Outro estudo que avaliou 13 pacientes, de ambos os sexos, com paralisia unilateral de prega vocal, antes e após a fonoterapia também obteve melhora na qualidade vocal após a fonoterapia em $69,2 \%$ dos pacientes, sendo que somente $15,3 \%$ não demonstraram melhora nos parâmetros perceptivo-auditivos ${ }^{21}$. Na pesquisa que analisou a voz de 40 indivíduos com paralisia unilateral de prega vocal antes e após a terapia fonoaudiológica e encontrou, do mesmo modo, melhora nos parâmetros grau geral $(G)$, soprosidade (B) e rugosidade $(R)^{3}$. Apesar do presente estudo não ter encontrado diferença estatisticamente significante do parâmetro perceptivo-auditivo de rugosidade (R) comparando-se os momentos pré e pós fonoterapia, há uma melhora deste parâmetro após o tratamento fonoaudiológico.

De acordo com a tabela 2, os traçados espectrográficos obtiveram melhora em $91,7 \%$ dos casos, sendo que os demais permaneceram estáveis $(8,3 \%)$. Os parâmetros que mais sofreram melhora após a alta fonoaudiológica foram aumento do número de harmônicos $(32,2 \%)$ e a diminuição do ruído $(24,2 \%)$. A diminuição da presença de ruído pode ser explicada devido a melhor coaptação glótica apresentada pelos pacientes, deste estudo, com paralisia unilateral de prega vocal após a fonoterapia, evidenciado no exame da laringe ao final do tratamento. A paralisia unilateral de prega vocal persistiu após a fonoterapia, porém o fechamento glótico apresentou melhoria, fato este devido à compensação pela prega vocal contralateral durante a coaptação glótica, e consequentemente menor escape aéreo que se configura como ruído na espectrografia. $\mathrm{O}$ aumento do número de harmônicos definidos se dá, do mesmo modo, devido à melhor coaptação glótica, possibilitando maior sonorização do ar. Tais achados são concordantes com a análise perceptivo-auditiva, uma vez que foi possível observar uma melhora nos parâmetros de soprosidade (B), também relacionado com uma melhor coaptação das pregas vocais.

A análise acústica demonstra quantitativamente aspectos mensuráveis do sinal sonoro e é utilizada para complementar a avaliação vocal subjetiva. Vários são os parâmetros acústicos obtidos nesta análise, sendo os mais comuns: a frequência fundamental, jitter, shimmer e proporção harmônico-ruído (PHR). Entretanto, de acordo com $^{30}$ sinais muito aperiódicos ou caóticos, não permitem mensuração confiável. Diante deste dado, não foram considerados os parâmetros da análise acústica desta pesquisa, uma vez que emissões de indivíduos com paralisia unilateral de prega vocal se configuram como aperiódicas.
Foram encontradas na literatura pesquisas que analisaram o comportamento de medidas acústicas a curto prazo em indivíduos com paralisia de prega vocal. Um estudo avaliou 40 indivíduos com paralisia unilateral de prega vocal antes e após a terapia fonoaudiológica e os valores acústicos de jitter\%, shimmer\% e PHR apresentaram melhora estatisticamente significante após a terapia vocal ${ }^{3}$. Outro estudo avaliou 13 pacientes, de ambos os sexos, com diagnóstico de paralisia unilateral de prega vocal, antes e após a terapia fonoaudiológica e, os valores médios de perturbação de frequência (PPQ), perturbação de intensidade (APQ) e presença de ruído (NHR) apresentaram-se menores ao final do tratamento, porém estes dados não foram estatisticamente significantes ${ }^{21}$.

Utilizando a análise do traçado espectrográfico em pacientes com paralisia unilateral de prega vocal, foram encontradas duas pesquisas, porém, com diferenças metodológicas desta pesquisa ${ }^{3,24}$.

O TMF é um teste usado rotineiramente para avaliação indireta da coaptação glótica à fonação. $O$ TMF encontra-se reduzido nas paralisias de prega vocal devido ao fechamento glótico incompleto e consequente fenda glótica. A tabela 4 evidencia diferença estatisticamente significante nos valores do TMF comparando-se os momentos pré e pósfonoterapia $(p=0,003)$. Este resultado concorda com os dados encontrados na pesquisa que avaliou a emissão sustentada das vogais /a/, /i/ e /u/ de 86 indivíduos adultos, de ambos os sexos, com paralisia unilateral de prega vocal e encontrou que a diminuição dos valores do TMF está associada ao maior ângulo de afastamento da PVP9 . Outro estudo que analisou a voz de 30 indivíduos, do sexo feminino, com paralisia unilateral de prega vocal antes e após a fonoterapia encontrou também aumento de 6,50 s para $11,15 \mathrm{~s}$ nos valores do TMF após a terapia fonoaudiológica ${ }^{25}$. O trabalho que avaliou vozes de 13 pacientes, de ambos os sexos, com paralisia unilateral de prega vocal observou, igualmente, melhora nos valores do TMF em $53,8 \%$ dos pacientes, apesar destes permanecerem abaixo dos padrões de normalidade ${ }^{21}$.

Os protocolos de qualidade de vida relacionados à voz têm sendo muito utilizados para mensuração do impacto e desvantagem da disfonia na vida do indivíduo ${ }^{21,23,31}$. Este estudo não conta com dados da auto-avaliação da voz, porém, pesquisas futuras que analisam o impacto da disfonia na qualidade de vida de indivíduos com paralisia unilateral de prega vocal são importantes 


\section{CONCLUSÕES}

Da avaliação de 12 pacientes com paralisia de prega vocal unilateral nos momentos pré e pósfonoterapia pode-se concluir que:

1) Os parâmetros perceptivo-auditivos que apresentaram melhora no momento pós-fonoterapia foram soprosidade $(B)$, grau geral da disfonia (G) e astenia (A);

2) Há melhora no traçado espectrográfico no momento pós-fonoterapia nos parâmetros de: aumento do número de harmônicos e diminuição do ruído;

3) Há aumento do TMF da vogal/a/ no momento pós-fonoterapia.

\begin{abstract}
Purpose: this study aims to analyze the objective and subjective evaluation of voice quality in a unilateral vocal fold mobility alteration on prior and post speech therapy. Method: this is a retrospective study through chart review, which analyzed the recorded voices of 12 individuals with otorhinolaringology diagnosis of unilateral vocal fold paralysis. The collected material was the sustained emission of the vowel /a/, and then connected speech. The voices before and after therapy were analyzed by using GRBASI scale. The parameters for spectrographic analysis were: shape of the spectrographic tracing, degree of browning of harmonics, stability of the tracing of harmonics, presence of noise, presence of harmonics, presence of sub-harmonics and harmonics definition. The measurement of the maximum phonation time for the vowel /a/ represented the highest value among three emissions. The obtained data were submitted to statistical analysis through Wilcoxon Test Ranking. Results: in the perceptual analysis, the parameter that has changed in the post-treatment was breathiness $(B)$ $(p=0.003)$, followed by dysphonia grade $(G)(p=0.004)$ and asthenia $(A)(p=0,01)$, and those results were statistically significant. As for the spectrogram, we found an improvement of the tracing in $91 \%$ of patients, and the changed parameters were: increase in number of harmonics (32\%) and reduction in noise (24\%). Measurement of the maximum phonation time of the vowel /a/ was higher in the postspeech therapy $(p=0.003)$. Conclusion: speech therapy treatment of unilateral vocal fold paralysis improved perceptual-hearing and spectrographic data and the maximum phonation time of voice.
\end{abstract}

KEYWORDS: Voice; Voice Disorders; Speech Therapy; Vocal Cord Paralysis; Speech Acoustics

\section{REFERÊNCIAS}

1. Rechtenwald S. Paralisias laríngeas: etiologia. CEFAC - Centro de Especialização em Fonoaudiologia Clínica. 1998; 1-19.

2. Dedivitis RA, Coelho LS. Paralisia de prega vocal em tireoidite subaguda. Rev Bras Otorrinolaringol. 2007;73(1):144.

3. Schindler A, Bottero A, Capaccio P, Ginocchio $D$, Adorni F, Ottaviani F. Vocal improvement after voice therapy in unilateral vocal fold paralysis. J Voice. 2008;22(1):113-8.

4. Duarte BB, Mikinev RM, Costa KC. Paralisia Bilateral em Abdução de Pregas Vocais como Manifestação de Câncer de Esôfago: Relato de Caso e Revisão de Literatura. Arquivos internacionais de Otorrinolaringologia. 2006;10(4):327-30.

5. Martins RGH, Braz JRC, Dias NH, Castilho EC, Braz LG, Navarro LHC. Rouquidão após
Intubação Traqueal. Rev Bras de Anestesiol. 2006;56(2):189-99.

6. Ada M, Isildak H, Saritzali G. Congenital vocal cord paralysis. J Craniofac Surg. 2010;21(1):273-4.

7. Steffen N, Vieira VP, Yazaki RK, Pontes P. Configuração das Pregas Vestibulares em Pacientes Portadores de Paralisia Unilateral de Prega Vocal. ACTA ORL/Técnicas em Otorrinolaringologia. 2006;24(3):121-5.

8. Rubin AD, Sataloff RT. Vocal fold paresis and paralysis: what the thyroid surgeon should know. Surg Oncol Clin. 2008;17:175-96.

9. Steffen LM, Moschetti MB, Steffen N, Hanayama EM. Paralisia unilateral de prega vocal: associação e correlação entre tempos máximos de fonação, posição e ângulo de afastamento. Rev bras otorrinolaringol. 2004;70(4):450-5.

10. Garrel R, Nicollas R, Chapus E, Ouaknine M, Giovanni A. Voice improvement in unilateral laryngeal 
paralysis during loud voicing: theoretical impact. Eur Arch Otorhinolaryngol. 2007;264:1201-5.

11. Yangali R, Moreno K. Parálisis vocal permanente: corrección quirúrgica con tiroplastia tipo I. An Fac med. 2008;69(1):52-5.

12. Finger LS, Cielo CA. Aspectos fisiológicos e clínicos da técnica fonoterapêutica de fonação reversa. Rev Bras Otorrinolaringol. 2007;73(2):271-7.

13. Roman G, Cielo CA. Particularidades da técnica fonoterapêutica de sons hiperagudos: revisão de literatura. Rev CEFAC. 2006;8(3):360-7.

14. Behlau M, Pontes P. Avaliação e tratamento das disfonias. São Paulo: Lovise; 1995.

15. Behlau M. Azevedo R, Pontes P, Brasil O. Disfonias funcionais In: Behlau M. Organizadora. Voz: O livro do especialista. Rio de Janeiro, Revinter, 2001.

16. Olavarría L, Albertz A, Tabilo C, Valdés P. Experiencia clínica en el manejo de parálisis cordales en posición abierta: tratamiento actual. Rev Hosp Clin Univ Chile. 2008;19(2):97-104.

17. Hoffman MR, Witt RE, Chapin WJ, McCulloch TM, Jiang JJ. Multiparameter comparison of injection laryngoplasty, medialization laryngoplasty, and arytenoid adduction in an excised larynx model. Laryngoscope. 2010;120(4):769-76.

18. Hirano M.- Clinical examination of voice. Wien; New York: Springer-Verlag; c1981.

19. Dejonckere PH, Leback J. Acoustic, perceptual, aerodynamic and anatomical correlations in voice pathology. ORL J Otorhinolaryngol Relat Spec 1996;58:326-32.

20. Côrtes MG, Gama AC. Análise comparativa dos parâmetros espectrográficos da voz antes e depois da fonoterapia [Monografia]. Belo Horizonte (Minas Gerais):UFMG; 2007.

21. Mangilli LD, Amoroso MRM, Nishimoto IN, Barros APB, Carrara-de-Angelis E. Voz, deglutição e qualidade de vida de pacientes com alteração de mobilidade de prega vocal unilateral pré e pós fonoterapia. Soc Brás Fonoaudiol. 2008:13(2):103-12.

22. Paseman A. Casper J. Colton R. Kelley R. The effects of head position on glottic closure in patients with unilateral vocal fold paralysis. $\mathrm{J}$ voice. 2003:18(2):242-7.

23. Kelchner LN, Stemple JC, Gerdeman B, Borgne WL, Adam S. Etiology, Pathophysiology, Treatment Choices, and Voice Results for Unilateral Adductor Vocal Fold Paralysis: A 3-Year Retrospective. J voice. 1999;13(4):592-601.

24. Hartl DM, Hans S, Vaissière J, Riquet M, Brasnu DF. Objective Voice Quality Analysis Before and After Onset of Unilateral Vocal Fold Paralysis. J Voice. 2001;15(3):351-61.

25. D'Alatri L, Galla S, Rigante M, Antonelli O, Buldrini S, Marchese M R. Role of early voice therapy in patients affected by unilateral vocalfold paralysis. The journal of Laryngology \& Otology, 2007:1-6.

26. Sataloff RT, Praneetvatakul P, Heuer RJ, Hawkshaw MJ, Heman-Ackah YD, Schneider SM, Mandel S. Laryngeal electromyography: clinical application. J Voice. 2010;24(2):228-34.

27. Tiago RSL, Munhoz MSL, Faria FP, Guilherme A. Aspectos histomorfométricos do nervo laríngeo superior. Rev bras otorrinolaringol 2002;68(2):157-65.

28. Riad MA, Kotby,MN. Mechanism of glottic in a model of unilateral

vocal fold palsy. Acta otolaryngol. 1995;115:311-3. 29. Shiotani A, Okubo K, Saito K, Fujimine T, Tomifuji M, Ikeda A, Araki K, Momoshima S. Injection laryngoplasty with calcium phosphate cement. Otolaryngol Head Neck Surg. 2009;140(6):816-21. 30. Titze IR. Workshop on acoustic analysis: summary statement. lowa City: National Center for Voice and Speech, University of lowa; 1995.

31. Kasama ST, Brasolotto AG. Percepção vocal e qualidade de vida. Pró-Fono R. Atual. Cient. 2007;19(1):19-28.

http://dx.doi.org/10.1590/S1516-18462010005000137

RECEBIDO EM: 14/05/2010

ACEITO EM: 01/09/2010

Endereço para correspondência

lara Barreto Bassi

Av. Bernardo de Vasconcelos, 2600/306,

Ipiranga - Belo Horizonte - MG

CEP: $31160-440$

E-mail: iara.bassi@hotmail.com 